\title{
A New Way to 3D Scan
} Photo-based Scanning Saves Time and Money

\author{
A White Paper by \\ Alan Walford, Eos Systems Inc.
}




\section{Introduction}

Do you need to create dense 3D point clouds and detailed surface models of physical objects, and do you want to do it in a cost-effective and efficient manner? A new class of 3D scanning technologies, called photo-based scanning, may be the solution you are looking for.

This new technology uses a standard off-the-shelf digital camera in combination with specialized software that analyzes the digital images - there is no additional hardware required. The new technology is called photo-based scanning, or photogrammetric scanning.

In this white paper we define 3D scanning, review the current methods for 3D scanning, introduce photo-based scanning, plus examine strengths and weaknesses of the various technologies.

\section{What is 3D Scanning?}

Many applications require digital 3D models of objects to: a) perform measurement (volume, length), b) further modify for production/prototyping, c) document or visualize, d) reverse engineer, or e) perform quality assurance.

If the object or scene is complex and a high level of surface detail is required, creating the digital 3D model using point-based measurement methods (such as CMMs, touch-probe digitizers, or point photogrammetry) may not work or may not be efficient. Instead, we want to capture a high density of 3D points in a regular grid on the surface in an efficient manner - this is 3D scanning. We 'scan' the surface and at regular intervals measure the 3D location of that point. If the intervals are small we get a dense and accurate representation of the surface in our digital 3D model.

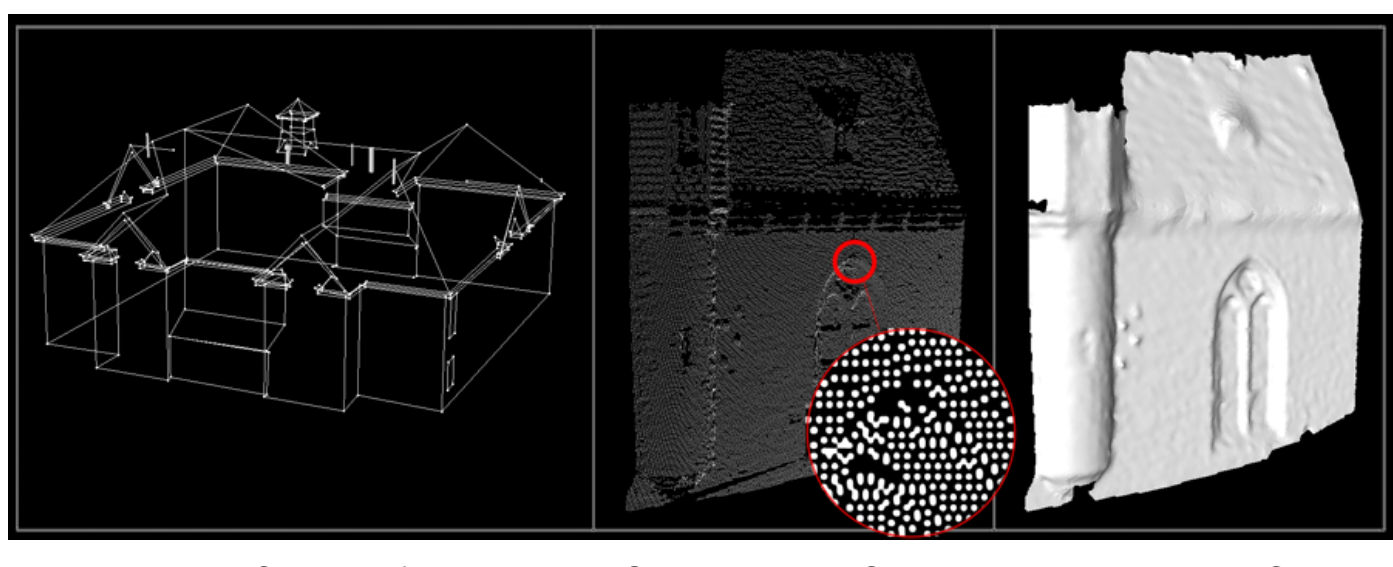

Typical CAD wireframe

Scanned Point Cloud

Meshed Point Cloud

A 3D point cloud by itself is typically not very useful. In this regard, some form of post processing is usually carried out. This post processing often involves filtering, hole filling, smoothing, meshing/triangulation and sometimes NURBS patch conversion. These functions are usually carried out by a separate software program, some of which are very expensive. 


\section{Typical Methods and Devices}

All 3D scanners use light rays in some form. A laser scanner sweeps a laser beam over the object and times how long it takes to return - this provides the distance from the scanner to every sampled point. Laser scanners take a number of forms: a) tripod mounted larger units for field work and large object/area scanning, b) desktop units for smaller objects, and c) hand-held or arm-based units also for small objects but with manual sweeping of the beam.

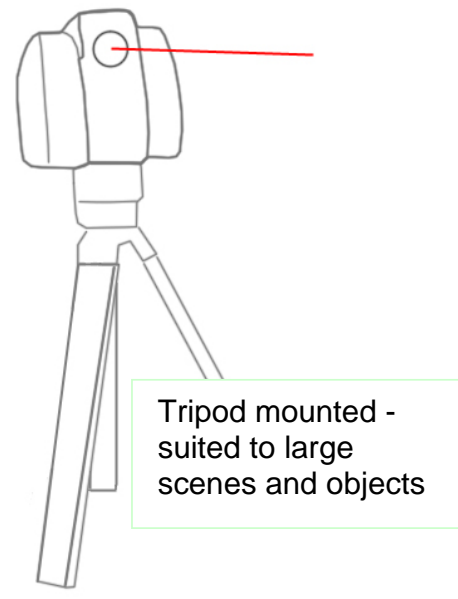

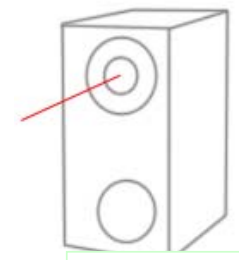

Desktop - suited to smaller and manufactured items

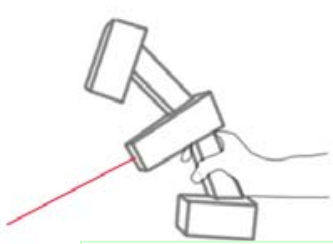

hand-held - suited to smaller manufactured items and those with complex shape

A white light scanner uses projected patterns (often stripes of different sizes) and one or more cameras to capture the reflected pattern. From the shape of the reflected pattern the white-light scanner can determine the positions of 3D points by triangulation principles.

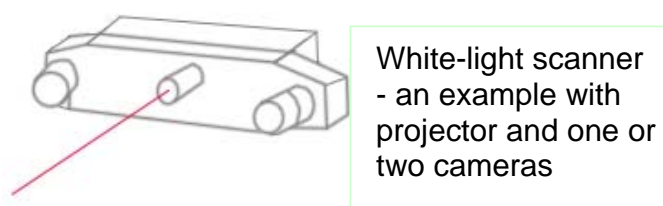

The new approach, called photo-based scanning, also uses light rays but in a passive way. Light is not sent out or projected from the scanner but instead the ambient light reflected off the object is captured by the camera from two or more positions. The following sections go into more detail.

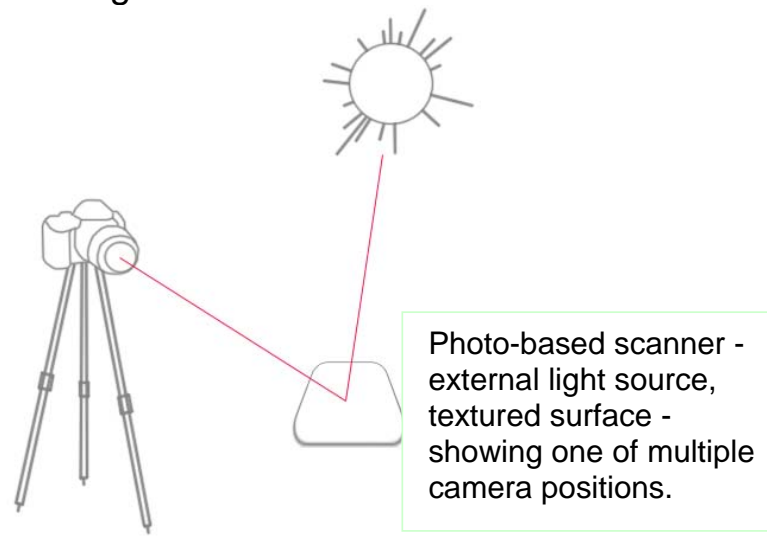


History of Photo-Based Scanning

Photo-based scanning has its historical feet in two camps: a) photogrammetric topographic Digital Elevation Model (DEM) creation, and b) computer vision stereo matching.

Early DEMs used in cartography were created manually, point-by-point, on an instrument called a stereo-plotter, using overlapping photographs taken from an airplane. In the 1980's this process started to become more automated with the use of film scanning and modern image matching techniques.

Stereo matching in computer vision started in the 1970's - made famous by Marr and Poggio and their study of human stereo vision. Humans detect depth using two eyes, so Marr and Poggio postulated that a similar mechanism could be developed for computer-based methods. Research since then has concentrated on improving the breadth and efficiency of matching across two images.

In 2008, the confluence of modern computers and development techniques brought us affordable close-range photo-based 3D scanning. This technique is based on the research and development of both camps - bringing the accurate measurement mindset from the photogrammetrists, and the advanced matching algorithm techniques from computer vision.

\section{How Does Photo-Based Scanning Work?}

Photo-based scanning is first based on a strong photogrammetric core. That means the system is capable of calibrating cameras, and is able to accurately solve for the position and orientation of the camera when it took the photos.

The photo-based scanning software then compares two photos on a small patch by patch basis. It is asking itself, "Is this small patch of image 1 like this small patch in image 2, or more like this other small patch in image 2?" It compares the patches across the images to find the best matches. When these optimal matches are found, the already-computed position and orientation information for the photographs is used to compute the location of that patch in 3D space.

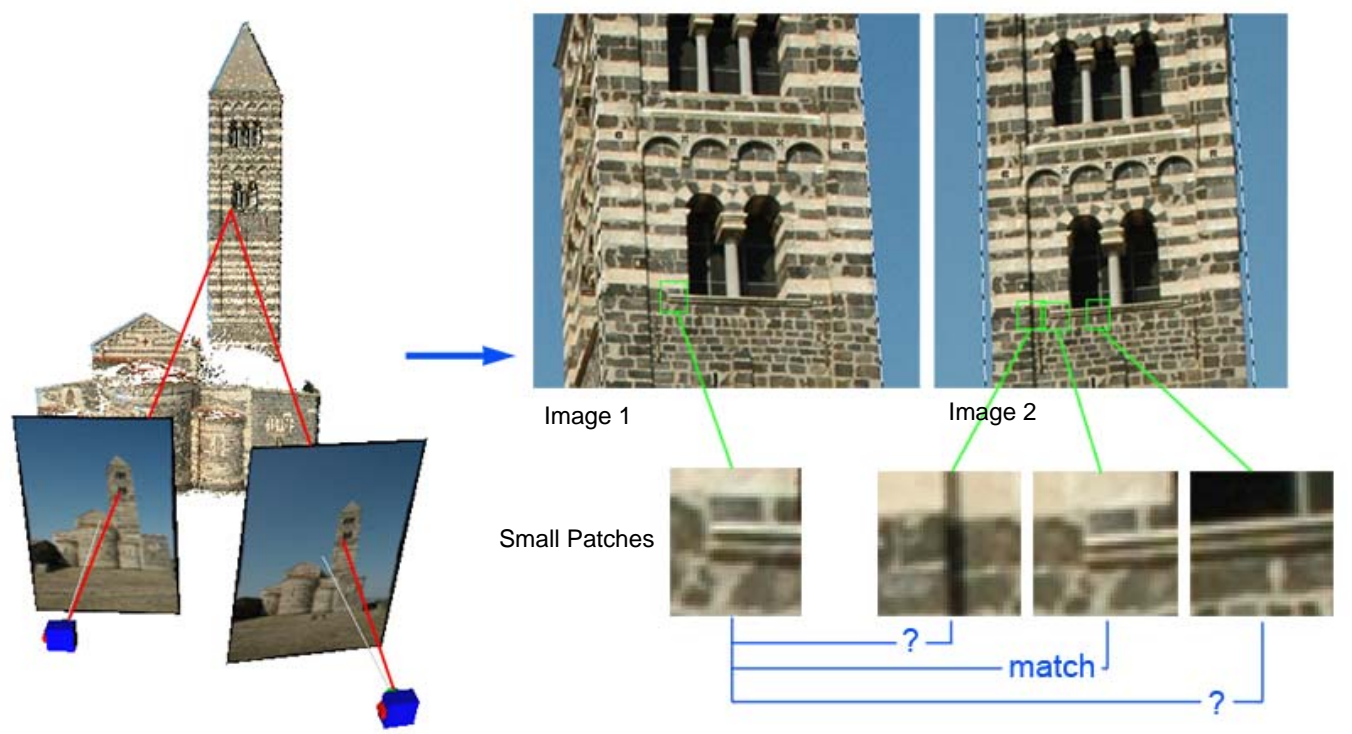


When a regular grid of patches is sampled in image 1 , and matched to the optimal image positions in image 2, the result is a dense cloud of 3D points. For this matching process to work, the surface being scanned must have a texture. That is, the surface cannot be blank (like a piece of paper or clean metal); instead it should have a random pattern (like carpet, a rock face, gravel, concrete, or even human skin).

\section{How Is Photo-Based Scanning Done?}

These are typical steps for executing a 3D scan with a photo-based scanning system:

1. Ensure the object or scene has a suitable pattern or texture on the surface.

2. Ensure good and consistent lighting during photography.

3. If there are no suitable, easily-identifiable points around the scene or object, place some high contrast targets.

4. Take two or more photographs of the object or scene - these are often taken as pairs with the pair of photographs being close to parallel but separated horizontally.

5. Load the photographs into the photo-based scanning software.

6. In the software perform the orientation step - where the software, using the targets or sharp point markings, solves where the camera was during photography.

7. Initiate the software's automated scanning/matching step on whole photos or selected sub-regions.

8. The resulting 3D point cloud can be filtered, meshed (to convert to a triangulated surface), or merged with other point clouds.

9. Export the result, perform measurements, or visualize with projected photo-textures.

\section{Examples of Photo-Based Scanning}

Below are a few representative examples of objects that have been scanned successfully with photo-based 3D scanning software. Each example shows a photograph of the object, and a 3D photo-scanner output (e.g. a view of the 3D model, triangulated surface, or point cloud). 


\section{Architecture}

This is a 3D scan of an Italian Basilica. The center 3D view shows individual points with RGB color extracted from the same photos used to create the scan this is not a surface but a dense point cloud. A single scan is shown - multiple scans would be used to generate a complete surface. The right 3D view shows 4 camera positions used to generate 2 scans.

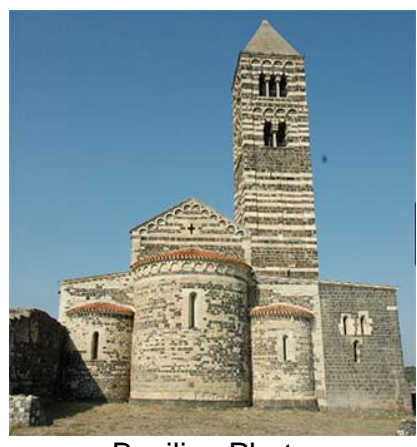

Basilica Photo

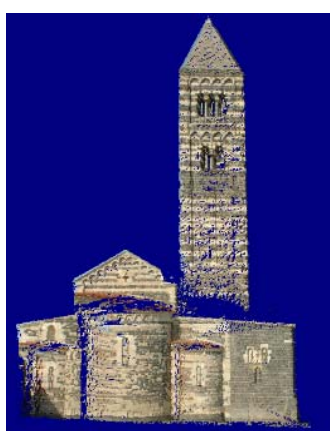

RGB Point Cloud

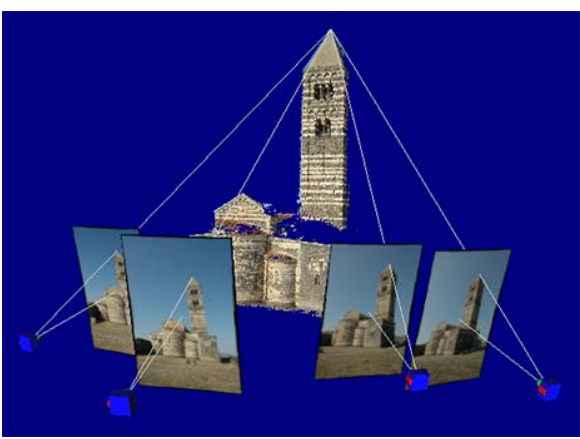

Showing photo positions

\section{Art / Preservation}

This is a combination model of CAD vector data (lower section) and dense 3D scan data (lion part). Some of the photo-based scanner packages allow this easy combination of modeling types from the same photographs.

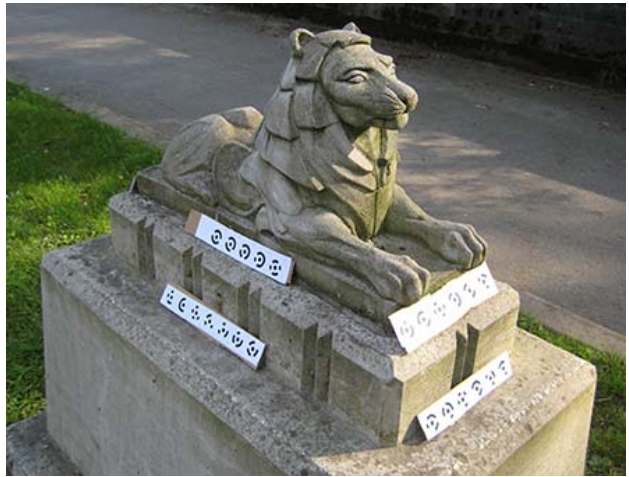

Lion Statue Photo

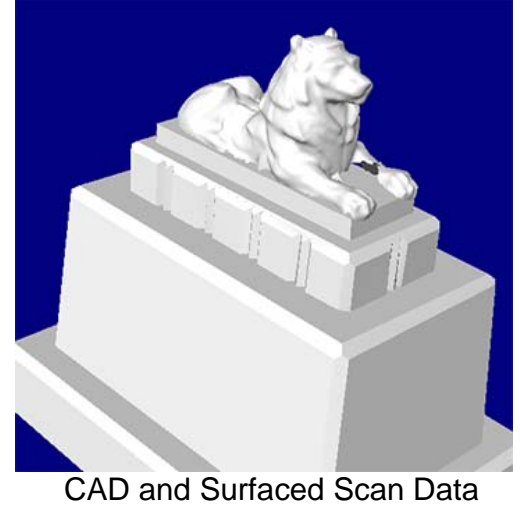

CAD and Surfaced Scan Data 


\section{Forensics}

This project is representative of a surface scan of an indent mark in a textured surface. In this case we have a shoe print in sand. Targets around shoe print automated the project setup. The 3D view is a detail shaded surface created from the point scan.

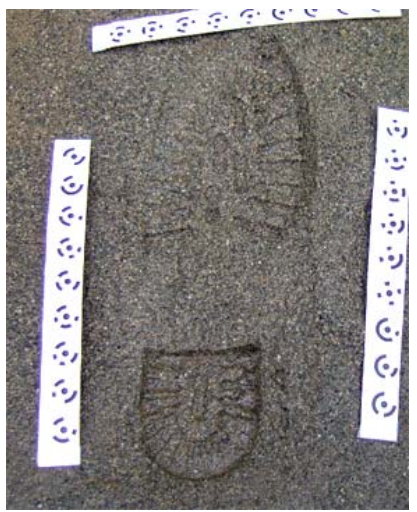

Shoe Print in Sand

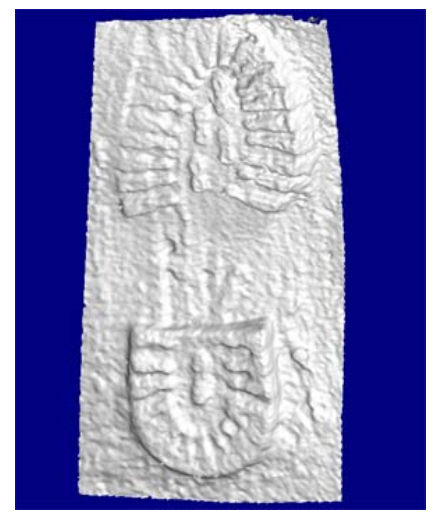

Shaded Model

\section{Geology / Civil Engineering}

This scan is of a small rock face showing sea erosion. The 3D scan results show the triangulated and filtered surface model (shaded) and height contours:

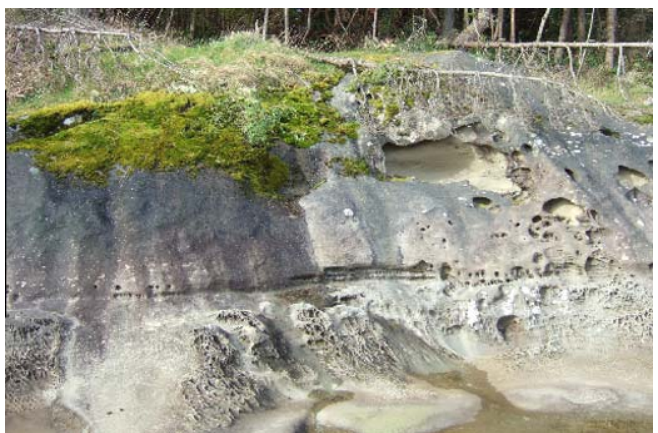

Eroded Rock Face Photo

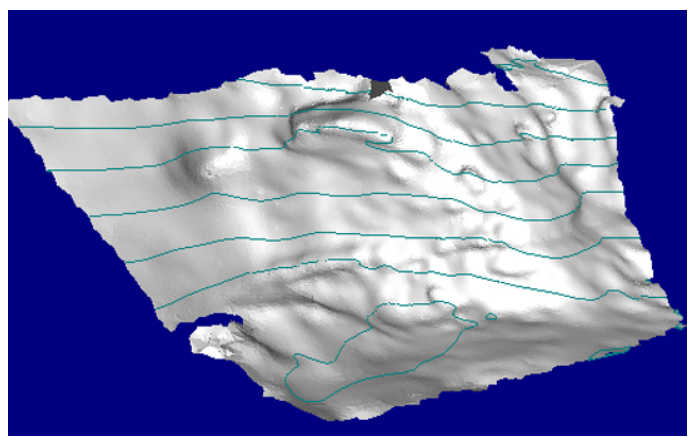

Scan Shaded Surface w/ Height Contours 


\section{Medical / Human Scanning}

Skin has a subtle texture, and human subjects add the complexity of movement, but with the correct settings and equipment, human skin (and clothing) can be successfully scanned with a photo-based scanner. Two examples are shown here - a back (as one might need for pre-op or scoliosis studies) and a face. The 3D view of the face shows a meshed point cloud with projected photo textures (this is a 3d model, not a photograph!).

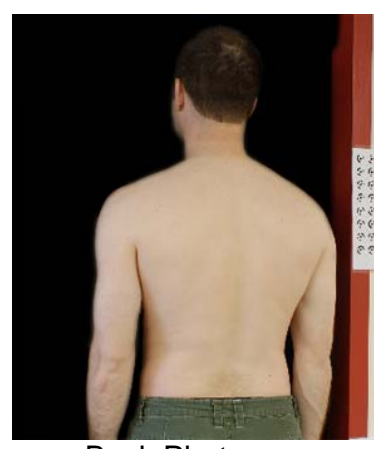

Back Photo

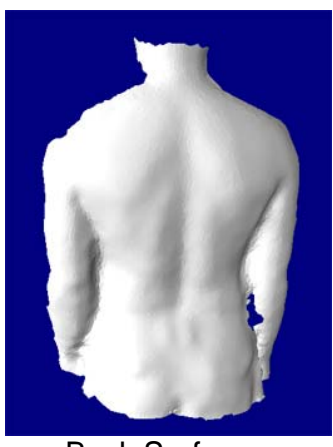

Back Surface

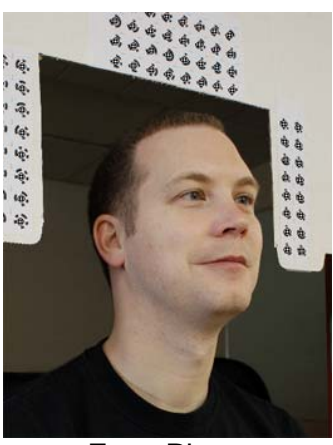

Face Photo

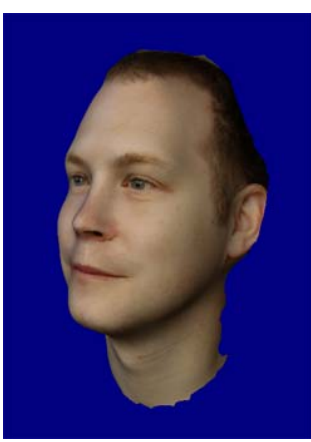

Face 3D Model

\section{Volume Measurement}

This project models a gravel stock pile (from aerial imagery). The 3D view shows a shaded surface model and height contours. An accurate volume measurement can be made from this surface model.

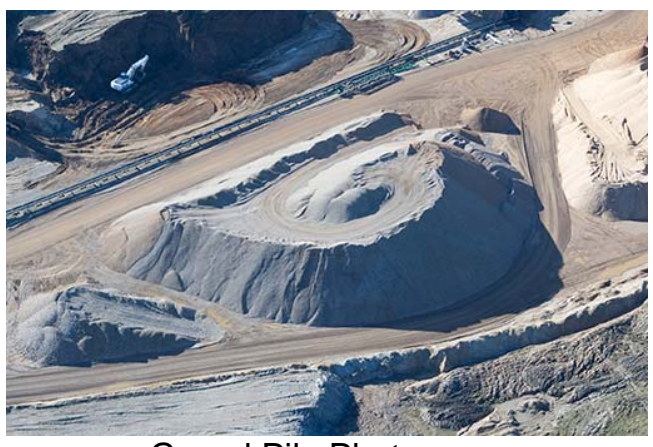

Gravel Pile Photo

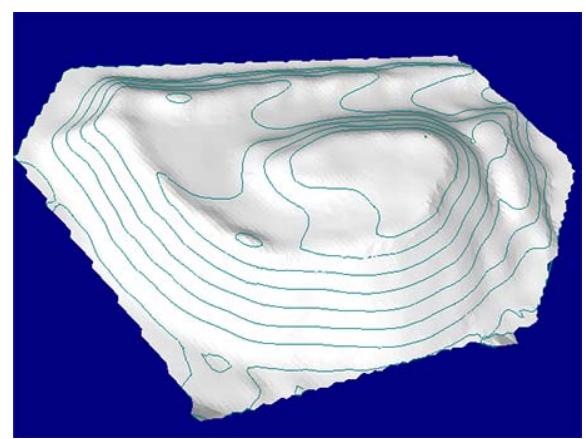

Extracted Surface Model w/ Contours 


\section{Comparing Photo-Based Scanning and Other Methods}

Here are some criteria for comparing 3D scanning methods:

- accuracy (of each solved 3D point)

- resolution (density of the point cloud)

- mobility of the system and instruments

- distance range ability

- time: time to set up, scan time, and processing time

- versatility / restrictions

- purchase cost

- on-going costs and maintenance

\section{Accuracy / Resolution}

Accuracy is how close each point's 3D position is to the true shape of the surface. Resolution is how close the 3D points are to each other. Accuracy varies over these methods with the desktop scanners or arm-based scanners tending to be the most accurate and the highest resolution in absolute terms. Note they are high-accuracy and high-resolution because they scan smaller areas.

A photo-based scanner's accuracy and resolution are affected by the resolution of the camera being used, the distance of the camera to the subject, and the nature of the texture and pattern on the surface. For any given surface and camera distance there is a natural resolution for a photo-based scan. Asking for a resolution above this generates extra noise (i.e. some inaccurate 3D points). The surface texture can't be changed but often the resolution can be improved by moving the camera closer or using a higher-resolution camera.

For a surface with a good texture pattern you can usually get one 3D sample per image pixel. For example, a 10MP camera viewing a $1 \mathrm{~m}$ object has each pixel viewing approximately $0.3 \mathrm{~mm}$ of surface. Here the resolution of the 3D sampling would be approximately $0.3 \mathrm{~mm}$. Stepping back so the camera now views a $10 \mathrm{~m}$ object, the point cloud resolution falls to $3 \mathrm{~mm}$.

The resolution of a laser scanner is fixed by mechanical limitations (how precisely they can encode angles of the laser projector). The resolution is in terms of angle and so the farther the subject, the lower the surface resolution. In this way the resolution of the imaging chip in the camera used in photo-based scanning is similar to the angle resolution of a laser scanner. A laser scanner is not affected as much by the surface texture as photo-based scanning is. The accuracy of a laser scanner is based on how accurate the electronics are for measuring time of flight or phase, and how accurate the encoders for angle are.

The accuracy of a hand held or arm-based laser scanners might be $0.2 \mathrm{~mm}$ (for a $10 \mathrm{~cm}$ object). A high-precision desktop scanner might have $0.01 \mathrm{~mm}$ accuracy (objects up to $1 \mathrm{~m}$ ). A large-area, tripod-mounted unit might have $5 \mathrm{~mm}$ accuracy (objects up to $50 \mathrm{~m}$ size). Note that there is much variation in laser scanners and these figures are only representative examples.

\section{Mobility}

When working in an office or laboratory hand-held laser scanners or desktop units remain in place once installed and mobility is not usually an issue. If they do have 
to be moved they are relatively small and pack in a suitcase-size case. The tripod-mounted, large-area scanners are mobile but do take up a few large, heavy and somewhat fragile cases. In a photo-based scanning system, all that travels is your digital camera; the ultimate in mobility. A notebook computer loaded with the photogrammetric scanning software may optionally be taken on site if initial results need to processed and viewed in the field. Otherwise, with a photo-based system, the photographs can be processed later at the office when it is more convenient.

\section{Distance Range}

The range of the scanner is how far the scanner can be from the object or surface and operate correctly. The range often correlates to the size of the object that can be scanned: the further back the scanner, the bigger the object that can be scanned. Desktop and hand-held laser scanners are designed for a smaller range (usually between $5 \mathrm{~cm}$ and $1 \mathrm{~m}$ ). Tripod-mounted scanners have a large range (usually $2 \mathrm{~m}$ to $50 \mathrm{~m}$ ). Photo-based scanning depends on the photographs - so if you can take a photograph of it, it can be scanned - giving photo-based scanning the widest range capability (from a penny to a mountain).

\section{Versatility and Restrictions}

Some scanners are restricted by the size of object (as described above in Distance Range). Photo-based scanning is not restricted by size but does require the surface to have a visible (visible in the photos taken) texture that is somewhat random. Random texture examples are carpet, rock, gravel, concrete, brick, skin, and fabric. Objects with little texture (metal parts, newly painted glossy surfaces, etc.) do not work as well with photo-based scanning. In addition photo-based scanning requires suitable lighting - so it cannot scan in the dark like a laser scanner without fixed artificial lighting.

\section{Time}

There are a number of time related criteria when considering 3D scanning. These are: a) the time to set up the instrument, b) the time to scan the object, and c) the time to process the data (usually back at the office).

\section{Time to set up}

The time to set up the scanner depends on the application and the scanner. For desktop applications, the scanner sits on a desk and is not moved. In this case the setup time is not relevant. For in-the-field applications, the large tripodmounted scanner can take some time to setup and calibrate, where as photobased scanning requires only that you turn your camera on and check your settings.

In addition, when doing scans from more than one position (which is a common requirement as scanners only capture one view from an instrument position and there will often be "occlusions" (i.e. unsighted areas)), the instrument has to be moved to new locations and its position relative to the other positions determined (so that multiple-scans can be married together in 3D). Note that hand-held and arm-based scanners are designed to scan from multiple positions and this is not an issue for them. Photo-based scanning determines the coordinate system of all photographs as part of the standard processing and so multiple-scans are inherently all placed in the same coordinate system without extra work. 
Time to scan

The time to scan the object can have a big impact on the type of object that can be scanned. Laser scanners and most white light scanners are not instantaneous and so require objects to be stationary during scanning and during instrument moves (to scan a different surface of same object). Some laser and white-light scanners are quick enough to scan a live human, but only with a great deal of cooperation by the subject. Photo-based scanning when done with a single camera has a similar problem with moving subjects but has a solution that other scanners do not have available to them. One configures multiple cameras that are synchronized so that the moving subject is captured at one instant in time. This allows a photo-based scanner to scan moving and shape-changing objects. You can cover multiple areas of the object at once by using more than two synchronized cameras. High-speed cameras allow the capture of very fast moving objects - not an option with laser or white-light scanners.

\section{Time to process}

Laser scanners usually produce their 3D point data in real-time. Some white-light scanners require computing to form the $3 \mathrm{D}$ data. Photo-based scanning does its 3D scanning as a computation - usually back at the office on your PC. To produce the raw point clouds photo-based scanning takes the most in-office computing time (producing 1000 to 5000 3D points per minute on a typical modern singlecore PC).

If multiple scans have been done (typically needed for a complete scan of most objects) then laser scan and white-light scan data will need some post processing to form a consistent coordinate system and match up the point clouds. This step is not needed with photo-based scanning.

Lastly, for all methods the point data often requires filtering, smoothing, hole filling and triangulation / meshing. These are all carried out in the office on the PC for all scanning methods.

\section{Costs}

There are two costs related to 3D scanning: a) the purchase or lease cost, and b) the maintenance costs. Most laser scanners, being fine mechanical instruments, will require periodic maintenance and calibration. Some white-light scanners are the same and others, being mostly electronics-based, are not. Photo-based scanners only require the maintenance of a typical digital camera (usually little to none). Calibration with photo-based scanners can be done in-house and the instrument does not need to be returned to the manufacturer.

A photo-based scanning system, being composed of a standard digital camera and software, has one of the lowest upfront costs. The camera and software together usually cost less than $\$ 3500$ us and if you have your own DSLR camera already, the cost to purchase is even less. There are some desktop laser scanners in the same price range, while larger scanners can cost tens or even hundreds of thousands of dollars.

In addition, software to do point cloud processing (filtering, smoothing, hole filing, and meshing) is an extra cost with most scanners (costing thousands to tens of thousand of dollars). Some photo-based scanners include point processing 
software integrated into the overall software solution. Photo-based scanning systems are the most cost effective overall.

\section{Technology Comparison}

Tripod-mounted laser scanners might do a better job than photo-based scanning in situations involving:

- poorly lit areas, night time scanning

- textureless surfaces (manufactured parts, newly painted surfaces, etc.)

- surfaces with large and abrupt depth changes

- surfaces at very shallow angle to the scanner

- processing time at the office must be a minimum

- very high densities and millions of points are required per scan

Desktop laser or structured light scanners might be better suited when the characteristics are:

- small objects with high detail and high density requirements

- textureless surfaces or a surfaces where texture is not dense or random

- where processing time at the office must be a minimum

Photo-based scanning might do a better job than laser or white-light scanners

where considerations include:

- equipment and maintenance costs

- wide variety of ranges and high versatility

- easy transport and low equipment weight

- quick field setup and minimal field time

- easy set up of multiple-scans in consistent coordinate systems

- scanning of moving subjects (with synchronized cameras)

\section{Conclusion}

Choosing the right scanning technology is, like most purchasing decisions, a blend of cost and function. With current options for 3D scanning, functional advantages do not necessarily come with high cost. Instead, selection by functionality may depend on the requirements of your specific applications, since all methods have strengths and weaknesses when compared to one another. The positive news is that 3D scanning systems are available at significantly different price points, some of which represent a realistic budget for almost any business.

\section{A Photo-Based Scanning Product}

A well-known photo-based scanning product is PhotoModeler Scanner by Eos Systems Inc. PhotoModeler Scanner is a cost-effective solution for 3D scanning if the application type has suitable characteristics. One needs just a standard digital camera, and the PhotoModeler Scanner software (runs on Windows and lists for \$2695us). The software also includes basic post-processing for point clouds (meshing/triangulation, filtering, hole filling and contouring) at no additional cost.

A unique and powerful aspect of PhotoModeler Scanner is that it includes all the vector/CAD-based and accurate $3 \mathrm{D}$ measurement tools of the base 
PhotoModeler product. This allows one to combine vector measurement with scanning within one project and with one set of photographs. This is well illustrated with the lion statute model shown in the examples section above. The top part (the lion) was 3D scanned, and bottom part (the base) was manually marked with lines, points and simple surfaces, all from one set of photos and all within PhotoModeler Scanner - resulting in a consistent model with no difficult merging or coordinate alignment processes required.

For further information about this product and to download a free product demo, please visit www.photomodeler.com.

\begin{abstract}
About the Author
Alan Walford graduated with Honors from the University of Waterloo with a Bachelor of Applied Science in Electrical Engineering and Computer Science (1984), and a Master's of Systems Design Engineering (1986). Alan is the founder and CEO of Eos Systems Inc., a pioneer in the development of closerange photogrammetry software. Photogrammetry is the science of extracting measurements and 3D data from photographs. Under Alan's management, Eos Systems has continuously held a leadership position in its market since its inception in 1990. Prior to Eos Systems, Alan held various software development and management roles in Atomic Energy Canada, Norpak, Raytheon Canada, MPR TelTech, and MacDonald Dettwiler. Alan is a successful entrepreneur and one of the world's leading experts in digital close-range photogrammetry.
\end{abstract}

